Results We identified 318 items of prescriptions for 61 patients an average of 5 items/patient, 68 patients without medication. Prevalence of $7.5 \%$ for unapproved drugs and $27.7 \%$ for off-label, and the more prevalent off-label use was regarding to age $-19.5 \%$. It was computed 57 medications - one patient used 10 off-label drugs during hospitalization. The prevalence of off-label uses was higher in infants $<35$ weeks $(p=0.00)$. Sepsis, malformation and extreme prematurity were the main causes to prescribe an off-label medication $(\mathrm{p}=0.00)$.

Conclusion Neonates exposed to off-label drugs in NICU had more severe disease, and because it is known that newborns, especially premies, use many drugs, it is necessary to prioritize research in the pharmacotherapy of this population so vulnerable.

\section{EFFECT OF ANESTHETIC USED IN LABOR ON TRANSCUTANEOUS BILIRUBIN LEVEL IN THE NEWBORN IN AN UNIVERSITY HOSPITAL IN BRAZIL}

doi:10.1136/archdischild-2012-302724.1643

1.2PDJH Nader, ${ }^{3,4} \mathrm{SS}$ Nader, ${ }^{2} \mathrm{H}$ Raymundo Chinazzo, ${ }^{2} \mathrm{DF}$ Dolvitsch. 'ULBRA, RS, ${ }^{2}$ ULBRA; ${ }^{3}$ Pediatrics, Universidade Luterana do Brasil, Canoas; ${ }^{4} H U$ ULBRA/Mãe de Deus, Porto Alegre, Brazil

Background and Aims Drugs administered on the mother can cross the placental barrier. The objective of the study is to analyze the effect of anesthetics used in caesarean section in the bilirubin level in neonates.

Methods This is a cross sectional study realized between August 2011 and March 2012, in rooming-in at Universidade Luterana do Brasil Hospital. All the babies born at term, without hemolytic disease, no fetal anomalies, apgar greater than 7 in the fifth minute and breastfeeding only. The transcutaneous bilirubin levels (TBLs) were obtained by the apparatus Dräger Jaundice Meter JM-103®. The TBLs variable was stratified according to the Bhutani nomogram. Were measured TBLs between 24 and 59 hours of life. This study was approved by the Ethics in Research.

Results From a total of 670 babies, 220(32.8\%) were delivered by caesarean section by epidural anaesthesia using bupivacaine (group A). In this group, $62.2 \%$ received morphine, $12.2 \%$ fentanyl and $36.8 \%$ sufentanil. The use of different opioids presented no significant association in TBLs, when compared with vaginally delivery. The TBLs in neonates of group A were lower than vaginally delivered ( $p=0.014)$. The use of bupivacaine decreased the risk of developing hyperbilirubinemia $(p=0.010)$. The use of ephedrine, $a$ vasoconstrictor, by $148(22 \%)$, only in group A, showed a lower risk of developing jaundice $(\mathrm{p}=0.010)$. The Buthani nomogram classified as low risk $530(79.1 \%)$ neonates.

Conclusions Babies born by cesarean have a higher probability to be classified as lower risk of developing hyperbilirubinemia $(p=0.015)$. Further studies are needed for definite results.

\section{SUCCESS OF A METHADONE TREATMENT PROTOCOL IN NEONATAL DRUG WITHDRAWAL FOLLOWING IN-UTERO EXPOSURE TO SUBSTANCES OF ABUSE}

doi:10.1136/archdischild-2012-302724.1644

${ }^{1} \mathrm{R}$ Schumacher, ${ }^{2} \mathrm{C} \mathrm{Ng},{ }^{1,3,4 \mathrm{~V}}$ Bhatt-Mehta. ${ }^{1}$ Pediatrics, University of Michigan Health System, Ann Arbor, MI; ${ }^{2}$ Pharmacy, Childrens Hospital of Philadelphia, Philadelphia, PA; ${ }^{3}$ Pharmacy; ${ }^{4}$ Center for Global Health, University of Michigan Health System, Ann Arbor, MI, USA

Background Neonatal abstinence syndrome (NAS) is often present following in-utero exposure to addictive substances. The Lipsitz tool is used to determine the severity of NAS. Failure to lower Lipsitz scores through supportive care results in pharmacological treatment with methadone, an opioid with an ideally suited long half-life.
Objectives To evaluate the effectiveness of a methadone treatment protocol for NAS.

Methods Neonates who received methadone treatment according to a preexisting treatment protocol were evaluated for treatment success defined as adherence to the methadone regimen with no residual signs of withdrawal. Data collected included: methadone dosages, Lipsitz scores, length of methadone treatment (LOT), and total length of stay (LOS).

Results Sixty subjects were included. The mean gestational age (GA) and birth weight (BW) were $36.8 \pm 3.03$ weeks and $2.79 \pm 0.63 \mathrm{~kg}$. All exhibited NAS within 72 hours of life. 59/60 (98.3\%) initiated treatment according to protocol. There was significant deviation from the protocol at 48 and 72 hours of treatment with $33 \%$ and $12 \%$ of the patients requiring more than the prescribed amount of methadone to control NAS. The mean (SD) total methadone exposure was $1.96 \pm 1.63 \mathrm{mg} / \mathrm{kg}$, LOT $11.66 \pm 9$ days and LOS $22.4 \pm 29.3$ days suggesting significant variability in response. No significant correlation was found between BW or GA and LOT.

Conclusion At diagnosis a protocol for treating NAS was closely followed. Despite a formal protocol there was substantial variability in total methadone exposure, LOT and LOS suggesting other contributory factors for the observed variability.

\section{VANCOMYCIN IN NEWBORNS: COMPARISON OF A STANDARD DOSE TO DOSING ADJUSTED FOR BIRTH GESTATION AND AGE}

doi:10.1136/archdischild-2012-302724.1645

D Duffy, M Gayfieva, MA Thomson. Imperial College Healthcare NHS Trust, London, UK

Background Vancomycin dosing regimens and individual pharmacokinetics vary in newborns. A time lag in achieving therapeutic levels often occurs with delays in treating sepsis or toxicity.

Aims To investigate initial therapeutic trough levels $(10-15 \mathrm{mg} / \mathrm{l})$ achieved by a standard dose ( $15 \mathrm{mg} / \mathrm{kg} /$ dose 12 hourly) for all newborns compared to new dosing accounting for birth gestation and postnatal age. ${ }^{1}$

Methods Admissions from March 2010- January 2012 were included. Data on gestation, age, initial therapeutic level (pre $4^{\text {th }}$ dose) and creatinine were analysed.

Results 111 treatment courses in 83 infants were evaluated. The new dosing increased the proportion of therapeutic levels $[18 / 68$ $(26 \%)$ vs.16/43 (37\%), $p=0.29]$, greatest in infants $\geq 29$ weeks at birth, $\geq 10$ days old (12\% vs. $66 \%)$. Toxicity decreased $[21 / 68(31 \%)$ vs. $9 / 43(21 \%), p=0.28]$. Sub-therapeutic levels were unchanged (40\% vs. $43 \%$ ). Creatinine was higher with toxic levels compared to therapeutic/low levels $(p<0.0001)$. No infants with creatinine $<100$ umol/1 had toxic levels. 18/27 (66\%) with toxic levels had a creatinine of $>100 \mathrm{umol} / \mathrm{l}$.

Conclusions Dosing accounting for birth gestation and age resulted in a greater proportion of therapeutic levels and less toxicity but sub-therapeutic levels remain frequent. We recommend large scale population studies to determine the optimal dosing strategy. Reference:

1. Wallis, Williamson. Arch Dis Child F\&N 2011; 96. $<29$ weeks at birth: $<10$ days: $20 \mathrm{mg} / \mathrm{kg} 18$ hourly; > 10 days: $15 \mathrm{mg} / \mathrm{kg} 12$ hourly.

$\geq 29$ weeks at birth: < 10 days: $15 \mathrm{mg} / \mathrm{kg} 12$ hourly; > 10 days: $15 \mathrm{mg} / \mathrm{kg} 8$ hourly.

\section{USE OF ADJUNCT CLONIDINE WITH PHENOBARBITAL TREATMENT FOR NEONATAL ABSTINENCE SYNDROME}

doi:10.1136/archdischild-2012-302724.1646

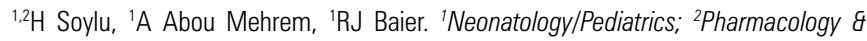
Therapeutics, University of Manitoba, Winnipeg, MB, Canada 